\title{
Weak localization competes with the quantum oscillations in a natural electronic superlattice: The case of $\mathrm{Na}_{1.5}\left(\mathrm{PO}_{2}\right)_{4}\left(\mathrm{WO}_{3}\right)_{20}$
}

\author{
Kamil K. Kolincio $\odot,{ }^{1,2}$ Olivier Pérez $\odot,{ }^{1}$ Enric Canadell $\odot,{ }^{3}$ Pere Alemany $\odot,{ }^{4}$ Elen Duverger-Nédellec, ${ }^{1,5}$ \\ Arianna Minelli ${ }^{\circ},{ }^{6}$ Alexei Bosak, ${ }^{6}$ and Alain Pautrat ${ }^{1}$ \\ ${ }^{1}$ CRISMAT, CNRS, Normandie Univ, ENSICAEN, UNICAEN, 14000 Caen, France \\ ${ }^{2}$ Faculty of Applied Physics and Mathematics, Gdansk University of Technology, Narutowicza 11/12, PL-80-233 Gdansk, Poland \\ ${ }^{3}$ Institut de Ciència de Materials de Barcelona (ICMAB-CSIC), Campus Bellaterra, E-08193 Bellaterra, Barcelona, Spain \\ ${ }^{4}$ Departament de Ciència de Materials i Química Fisica and Institut de Química Teòrica i Computacional (IQTCUB), \\ Universitat de Barcelona, Martí i Franquès 1, E-08028 Barcelona, Spain \\ ${ }^{5}$ Charles University, Faculty of Mathematics and Physics, Condensed Matter Department, Ke Karlovu 3, CZ-121 16 Praha 2, Czech Republic \\ ${ }^{6}$ ESRF - The European Synchrotron, 71, Avenue des Martyrs, F-38000 Grenoble, France
}

(Received 20 December 2019; accepted 6 April 2020; published 30 April 2020)

\begin{abstract}
We report an investigation of the combined structural and electronic properties of the bronze $\mathrm{Na}_{1.5}\left(\mathrm{PO}_{2}\right)_{4}\left(\mathrm{WO}_{3}\right)_{20}$. Its low-dimensional structure and possible large reconstruction of the Fermi surface due to charge density wave instability make this bulk material a natural superlattice with a reduced number of carriers and Fermi energy. Signatures of multilayered two-dimensional (2D) electron weak localization are consequently reported, with an enhanced influence of quantum oscillations. A crossover between these two antagonistic entities, previously observed only in genuine low-dimensional materials and devices, is shown to occur in a bulk crystal due to its hidden 2D nature.
\end{abstract}

DOI: 10.1103/PhysRevB.101.161117

The unfading interest in low-dimensional conducting materials originates from their remarkable electronic properties. A high critical temperature in superconductors [1,2], improved thermoelectric properties [3-6], and instability of the electron gas into a charge density wave (CDW) $[7,8]$ are consequences of a pronounced structural and electronic anisotropy. In particular, quasi-one- or two-dimensional (1D or 2D) conductors exhibit a partial Fermi-surface (FS) reconstruction at a CDW transition, accompanied by a strong reduction of free-electron density, an increase of the remaining carrier mobilities, and enhanced magnetoresistance $[9,10]$.

In addition, quantum corrections may strongly impact the low-temperature transport properties. The weak localization (WL) phenomenon is a well-known example. Another characteristic of low-temperature magnetoresistance effects in metals, especially of those with a high carrier mobility, is the existence of Shubnikov-de Haas $(\mathrm{SdH})$ quantum oscillations. They originate from the quantization of the electron density of states into Landau levels when exposed to a magnetic field, and are observed in high-purity samples in the limit $\omega_{c} \tau \gg 1$, where $\omega_{c}=\frac{e B}{m^{*}}$ is the cyclotron frequency, $m^{*}$ the effective mass of carriers, and $\tau$ the elastic scattering time. $\mathrm{WL}$ and $\mathrm{SdH}$ effects are a priori antagonistic because they are respectively favored and limited by disorder. However, due to the decrease of the WL contribution with magnetic field and with the restricted condition $\omega_{c} \tau \leqslant\left(k_{F} \ell\right)^{-1}\left(k_{F}\right.$ is the Fermi momentum and $l$ the electronic mean free path), a crossover can be observed between these two quantum processes, whose magnetic field range has been related to interaction effects [11]. This crossover has been previously observed in rare systems showing strong $2 \mathrm{D}$ characteristics: $2 \mathrm{D}$ electron gas heterostructures with high mobilities [12], quantum wells $[11,13,14]$, and graphene $[15,16]$. We show here that both WL and $\mathrm{SdH}$ are observed in a single crystal whose 2D character arises from the carrier confinement in conducting layers with a thickness of a few atomic planes. The sample under study belongs to the monophosphate tungsten bronze (MPTB) family $A_{x}\left(\mathrm{PO}_{4}\right)_{2}\left(\mathrm{WO}_{3}\right)_{2 m}(A=\mathrm{K}, \mathrm{Na}$, or $\mathrm{Pb})$. In this family, carriers originate from the $\mathrm{PO}_{4}$ groups, and are delocalized in the middle of $\mathrm{WO}_{3}$ layers, giving a pronounced anisotropic electronic structure $[17,18]$. The carrier density can be tuned by changing $m$ due to an increase of unit cells for constant carrier numbers and/or by the insertion of $A$ cations with a metallic character [19]. These $A$ cations are inserted in hexagonal tunnels to give $A_{x}\left(\mathrm{PO}_{4}\right)_{2}\left(\mathrm{WO}_{3}\right)_{2 m}$ [20]. Supplemental interest in this bronze family is that most of the investigated members have shown CDW transitions that have been explained using the hidden nesting concept [21,22].

$\mathrm{X}$-ray diffraction investigations performed at room temperature (RT) reveal that the whole pattern can be described using a monoclinic cell $[a=6.5483(6) \AA, b=5.2893(5) \AA$, $\left.c=18.0789(15) \AA, \beta=94.7000(4)^{\circ}\right]$ and the space group $P 2_{1} / \mathrm{m}$; these cell parameters are in agreement with those reported for $\mathrm{Pb}_{x} \mathrm{P}_{4} \mathrm{~W}_{20} \mathrm{O}_{68}$ on the basis of a pattern matching analysis performed on the powder diffraction data [23]. The final agreement factor for our data is $4.7 \%$ and the refined chemical composition is $\mathrm{Na}_{1.49(8)} \mathrm{P}_{4} \mathrm{~W}_{20} \mathrm{O}_{68}$; $\mathrm{Na}$ sites are partly occupied. For details of the data collection, refinement, and the atomic structure parameters, see the Supplemental Material [24].

A projection along the $\mathbf{b}$ axis is shown in Fig. 1(a). The main structural characteristics expected for the MPTB 

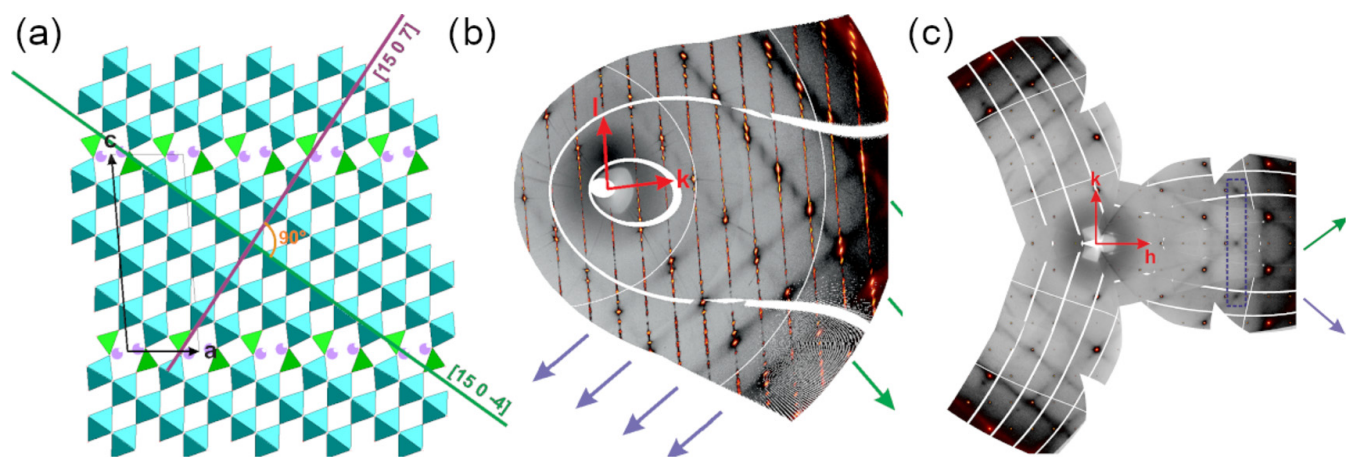

FIG. 1. (a) Projection along $\mathbf{b}$ of the structure of $\mathrm{Na}_{1.5} \mathrm{P}_{4} \mathrm{~W}_{20} \mathrm{O}_{68}$. Blue octahedra are $\left(\mathrm{WO}_{6}\right)$, green tetrahedra are $\left(\mathrm{PO}_{4}\right)$, and purple circles are $\mathrm{Na}$ atoms. The [15 07 7 ] and [ $150-4]$ directions are showing the short and long direction $\left(\mathrm{WO}_{6}\right)$ chains; they are $90^{\circ}$ oriented. $(\mathrm{b})(0 \mathrm{kl})^{*}$ and (c) $(h k 0)^{*}$ planes assembled from the frames collected at $130 \mathrm{~K}$ on the ID28 beamline at ESRF. Two directions of diffuse scattering are shown by green and blue arrows. Intensifications of the diffuse signal are observed at the intersection of the two types of diffusion scattering for $\frac{1}{2} \mathbf{a}^{*}$ (see blue dashed rectangle).

family in the fundamental state are observed. Slabs of the $\left(\mathrm{WO}_{6}\right)$ edge-sharing octahedra alternate with slices of $\left(\mathrm{PO}_{4}\right)$ tetraehdra; at the junction, hexagonal tunnels host $\mathrm{Na}$ atoms. Two orthogonal directions [ [15 07 7] and [15 0 - 4] can be highlighted; they correspond to chains of ten and five edge-sharing $\left(\mathrm{WO}_{6}\right)$ octahedra, respectively [see Fig. 1(a)].

To check the possibility of a CDW transition, full data collections were performed at RT and $130 \mathrm{~K}$ on the ID28 beamline at ESRF synchrotron [25]. There is no evidence for an additional scattering signal at RT but at $130 \mathrm{~K}$ diffuse scattering can be observed on different experimental frames. An accurate study of the $(h k 0)^{*}$ plane [see Fig. 1(c)] reveals an enhancement of the diffuse signal at the intersection of two diffuse planes for the $\frac{1}{2} \mathbf{a}^{*}$ wave vector. An analysis of the shift between the positions of the diffuse scattering in subsequent $(0 k l)^{*},(1 k l)^{*}$, and $(2 k l)^{*}$ reciprocal layers reveals that the diffuse sheets intersecting them run along the direction [15 07 7 $]$ (see Supplemental Material [24] for details).

The planes running along [15 07 7 $]$ are perpendicular to $\left[\begin{array}{lll}-7 & 0 & 15\end{array}\right]^{*}$, but $\left[\begin{array}{lll}-7 & 0 & 15\end{array}\right]^{*}$ and $\left[\begin{array}{lll}15 & 0 & -4\end{array}\right]$ fortuitously correspond to the same direction (see Supplemental Material [24]), the direction of the chain of ten octahedra. Then the observation of the diffuse scattering can be correlated with an ordering within the longest $\left(\mathrm{WO}_{6}\right)$ chain but with a loss of order both along $\mathbf{b}$ and along [15 $0-4]$, i.e., with a loss of order with the adjacent chains. The existence of diffuse scattering planes evidences the 1D ordering, possibly associated with CDW. Nevertheless, the absence of their condensation to sharp reflections shows that this ordering shows at $T=130 \mathrm{~K}$ a moderate coherence length, instead of establishing a longrange order.

Having established the crystallographic parameters, we studied the electronic structure of $\mathrm{Na}_{1.5}\left(\mathrm{PO}_{2}\right)_{4}\left(\mathrm{WO}_{3}\right)_{20}$. The results of band calculations performed with the generalized gradient approximation (GGA) (see Supplemental Material for details [24]) are shown in Fig. 2. There are large similarities between the studied compound and other MPTB members showing CDW $[21,26]$. As seen in Fig. 2(a), three bands are crossing the Fermi level resulting in a FS composed of three quasi-1D elements: three pairs of parallel planes running along $\left(a^{*}+b^{*}\right),\left(a^{*}-b^{*}\right)$, and $b^{*}$ directions with almost no dispersion along $c^{*}$ as shown in Figs. 2(a) and 2(c). While the presence of similar flat regions in MPTB hosting CDW gives a rise to hidden $1 \mathrm{D}$ nesting [21,26], their almost perfect alignment in $\mathrm{Na}_{1.5}\left(\mathrm{PO}_{2}\right)_{4}\left(\mathrm{WO}_{3}\right)_{20}$ additionally enhances the susceptibility for such a scenario. The relevance of this mechanism is additionally supported by the calculation of a Linhard function [Fig. 2(d)]. In particular, three very intense features near the $a^{*} / 2$ wave vector suggest that large parts of the Fermi surface are susceptible to destruction by either an inherent instability of the Fermi surface or coupling with another type of instability of the lattice with this wave vector.

Figure 3(a) shows the thermal variation of resistivity $\rho$ of a $\mathrm{Na}_{1.5}\left(\mathrm{PO}_{2}\right)_{4}\left(\mathrm{WO}_{3}\right)_{20}$ single crystal. Metallic behavior $\left(\frac{d \rho}{d T}<0\right)$ is observed from $400 \mathrm{~K}$ down to $10 \mathrm{~K}$, where a smooth increase of resistivity appears. The electronic resistivity follows a $-\ln T$ variation at low temperature, and this experimental observation suggests a WL contribution. The expression of the WL correction to conductance for noninteracting electrons is [27]

$$
\Delta G=\frac{p e^{2}}{2 \pi^{2} \hbar} \ln \frac{T}{T_{0}}
$$

where $p$ describes the temperature dependence of the inelastic scattering rate $\tau_{i} \propto T^{-p} . p$ is equal to 1 for phase breaking dominated by Coulomb interactions and to 3 for electron-phonon scattering [28]. Fitting the low-temperature part gives $\frac{d G}{d \ln T}=0.0517 \Omega^{-1}$, to compare with $\frac{e^{2} p}{2 \pi \hbar}=1.23 \times$ $10^{-5} \Omega^{-1}$ expected for a $2 \mathrm{D} \mathrm{WL}$ contribution. The large difference between these values can be explained if our sample is considered as a superlattice of $N$ independent and noninteracting 2D layers, giving rise to a multilayered $\mathrm{WL}$ effect [29-33]. Dividing the measured conductivity by the theoretical WL conductivity of a single layer gives $N \approx$ 4300 , considering a value $p=1$, as expected for electron scattering in $d$-electron systems. Multiplying $N$ by a thickness of the W-O conducting layer-approximately equal to the $c$ lattice constant-we obtain $\approx 10 \mu \mathrm{m}$, comparable to the thickness of the sample. The weak disorder causing WL can originate from a weakly nonuniform distribution of $\mathrm{Na}$ atoms in the tunnels [22], stacking faults reported for MPTB with 

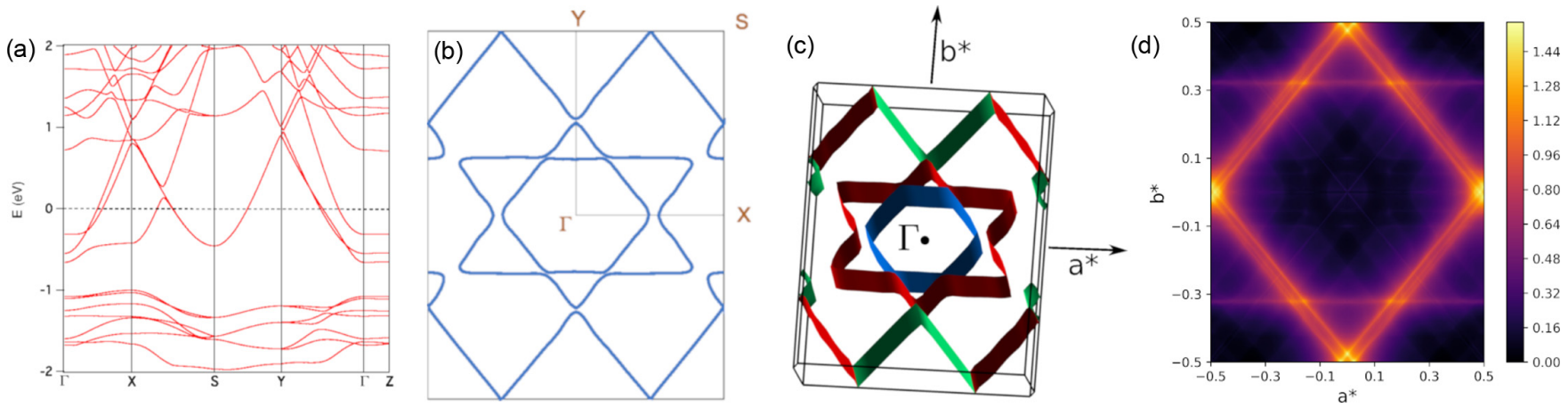

FIG. 2. (a) Electronic band structure calculated for $\mathrm{Na}_{1.5}\left(\mathrm{PO}_{2}\right)_{4}\left(\mathrm{WO}_{3}\right)_{20}$. Zero energy corresponds to the Fermi level and the different points are defined as $\Gamma=(0,0,0), X=(1 / 2,0,0), Y=(0,1 / 2,0), S=(1 / 2,1 / 2,0)$, and $Z=(0,0,1 / 2)$, respectively, in the units of the reciprocal vectors. (b) Section of the Fermi surface for $c^{*}=0$. (c) $3 \mathrm{D}$ representation of the Fermi surface. (d) Calculated Lindhard function.

a high $m$ parameter [34], or can be contributed by the short order of the charge density wave state.

Since other mechanisms can lead to a logarithmic increase of resistivity at low temperature (Kondo scattering, a strong Coulomb interaction), we analyzed the magnetoresistance $\left[\mathrm{MR}=\frac{\rho(B)-\rho(0)}{\rho(0)} \times 100 \%\right]$ in order to confirm the underlying mechanism. The low-temperature magnetoresistance depicted in Fig. 3(b) is nonmonotonic. It is negative for moderate $B$, and upon increasing the magnetic field, it shows a minimum followed by a notable increase. For $T \leqslant 18 \mathrm{~K}$, large oscillations of the resistance can be observed in the increasing part of $\operatorname{MR}(B)$.

As a strong argument for WL, we observe a negative MR for $B$ perpendicular to the conducting plane $\left(\theta=0^{\circ}\right)$ but it disappears in the parallel configuration $\left(\theta=90^{\circ}\right)$ - see the inset of Fig. 3(b). This is precisely what is expected in the 2D WL regime, where the orbital effect responsible for the MR is suppressed for parallel fields [35]. The low-field magnetoconductance $\Delta G=G(B)-G(0)$ in the WL regime can be described by the Hikami-Larkin-Nagaoka (HKN) expression $[28,36]$,

$$
\Delta G=\frac{e^{2}}{2 \pi^{2} \hbar}\left[\psi\left(\frac{1}{2}+\frac{1}{A}\right)+\ln (A)\right],
$$

where $\psi$ is the digamma function and $A=\frac{4 e D \tau_{i} B}{\hbar}$. To show the increase of magnetoconductance in the magnetic field, we have quantified and removed the classical $\sim B^{2}$ magnetoresistance component. Then, we fit the low-field curves with the WL expression considering the number of 4300 independent layers as found from the thermal dependence of conductivity. As shown in Fig. 3(c) the fits show good agreement with the experimental data. The diffusion length of localization (Thouless length) $L_{\phi}=\left(D \tau_{i}\right)^{1 / 2}$ was extracted from the fitting parameter and reported in Fig. 4(a). It is worth noting that $L_{\phi}$ is larger than the thickness of the conducting layer and approximately equal to the periodicity along the $c$ axis, confirming the regime of 2D coherence. When increasing the magnetic field, the dephasing regime emerges for $L_{B}=\left(\frac{2 \hbar}{e B_{\phi}}\right)^{1 / 2} \geqslant L_{\phi}$. The effect of the magnetic field is then no longer relevant for WL and a negative contribution disappears. We have compared the value of $B_{\phi}$ extracted from the fitting with the condition $L=L_{B}$ and the magnetic field value $B^{*}$ where the $G(B)$ ceases to grow with $B$ and where quantum oscillations immediately emerge. We find that these values are very close to each other (for example, for 4,8 , and $10 \mathrm{~K}$ the values obtained from the fits yield 5.27, 5.36, and 5.49 T, respectively, to compare with the observed $B^{*}=5.45,5.31$, and $5.53 \mathrm{~T}$, respectively). For the lowest temperatures, $L_{\phi}$ ceases to grow as the temperature is lowered, and even drops for $1.8 \mathrm{~K}$, where the negative MR term also starts to decrease. A plausible explanation for this effect is the further crossover from weak localization to weak antilocalization (WAL) $[37,38]$ due to strong spin-orbit effects (a)

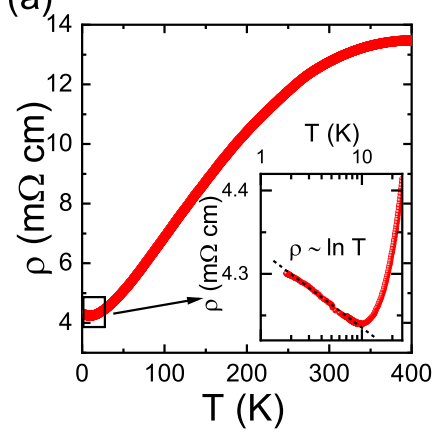

(b)

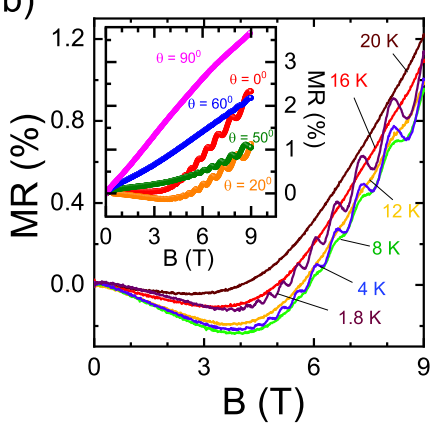

(c)

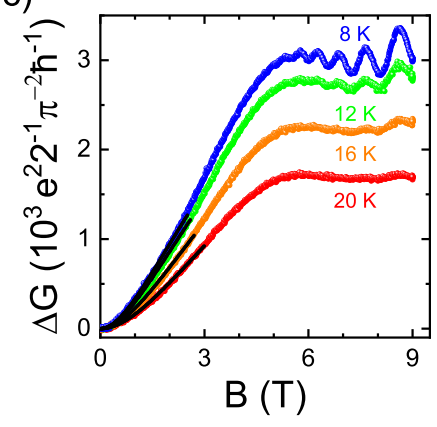

FIG. 3. (a) In-plane resistivity vs temperature. Inset: Expanded view of the low-temperature region shown with a logarithmic horizontal scale. The dashed line is a guide for the eye. (b) Magnetoresistance as a function of magnetic field applied parallel to the $c$ axis. Inset: MR $(B)$ plots at $T=1.8 \mathrm{~K}$ for various $\theta$ angles between the magnetic field direction and the $c$ axis. (c) Magnetoconductance vs magnetic field for selected temperatures. The red curves show the data fits with Eq. (2). 

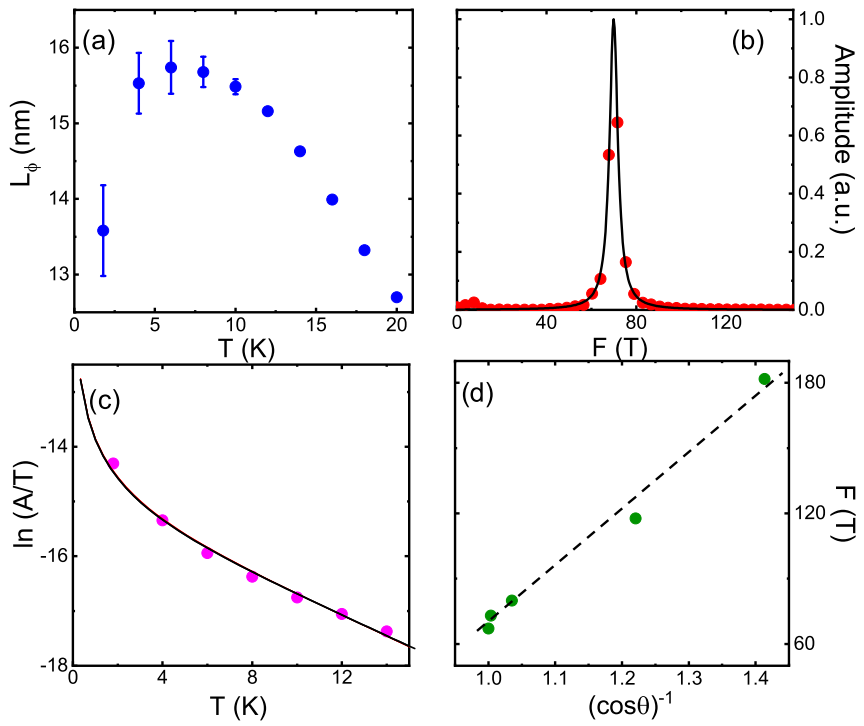

FIG. 4. (a) Temperature variation of the localization length, obtained from the magnetoconductance fits with Eq. (2). (b) Power spectrum of the oscillatory component of magnetoresistance. The solid line shows the Lorentzian fit to the experimental data. (c) The geometrical dependence of the oscillation frequency. The dashed line is a guide for the eye. (d) Thermal variation of the oscillation amplitude.

observed in tungsten-rich compounds [39,40]. This scenario is also supported by the emergence of sharp cusplike features in the low-field limit of magnetoresistance (see Supplemental Material), which is characteristic for the WAL regime [27,41]. A further examination of this term, to obtain a reliable HKN fit with the introduction of the antilocalization component, will require the transport measurements at temperatures below $1.8 \mathrm{~K}$

The oscillations in magnetoresistance emerge immediately after the negative WL term is suppressed, as evidenced in Fig. 3(c). They are periodic as a function of $1 / B$, as expected for Shubnikov-de Haas quantum oscillations. We have used the Lifshitz-Kosevich (LK) theory to extract the electronic parameters [42]. The Fourier transform of the oscillating part of magnetoresistance gives the power spectrum shown in Fig. 4(b). A peak can be observed at the frequency $F_{1}=69 \mathrm{~T}$ for $\theta=0^{\circ}$. This value yields the area $A_{1}$ of an extremal Fermi-surface cross section that is perpendicular to $B$, through the Onsager relation [43] $A_{i}=2 \pi e F_{i} / \hbar$. It corresponds to a FS cross section $A_{1}=0.68 \mathrm{~nm}^{-2}$, i.e., to a very small part $(\approx 0.5 \%)$ of the first Brillouin zone, estimated to be $110 \mathrm{~nm}^{-2}$. A plausible explanation for the presence of such a small pocket is the Fermi-surface decomposition associated with the charge density wave. This scenario is supported by the x-ray diffuse scattering and electronic structure calculations showing a potential for strong nesting around $a^{*} / 2$, which most likely occurs at high temperatures as for other high $m$ values of the MPTB family [44]. The temperature dependence of the quantum oscillation amplitude is depicted in Fig. 4(c). It can be related to the effective mass of quasiparticles $m^{*}$ through the temperature damping factor. We find $m^{*}=0.83 m_{e}$, with $m_{e}$ being the free-electron mass, indicating that the electron-electron interactions are moderate, as theoretically predicted for the WL-SdH crossover [11]. The field dependence of the quantum oscillations allows one to estimate the Dingle temperature $T_{D} \approx 6 \mathrm{~K}$, corresponding to an electronic mean free path $l \approx 62 \mathrm{~nm}$. Since the Fermi momentum is $k_{F} \approx\left(A_{1} / \pi\right)^{1 / 2}=0.47 \mathrm{~nm}^{-1}$, it leads to $k_{F} l \approx$ $29 \gg 1$, confirming the diffusive regime $[45,46]$. The angle $\theta$ between $B$ and the $c$ axis was varied from 0 to $90^{\circ}$ and the $\mathrm{SdH}$ oscillations can be observed within an experimental resolution up to $\theta \approx 50^{\circ}$. The frequency of the $\mathrm{SdH}$ oscillations clearly shifts to larger values as $\theta$ is increased, with an approximate $1 / \cos \theta$ variation, as depicted in the inset of Fig. 4(b). This is expected for quasi-2D Fermi-surface topology and is also consistent with the angular dependence of magnetoresistance shown in the inset of Fig. 3(b). For $B$ perpendicular to the conducting plane, MR initially attenuated by the negative contribution steeply increases with $\sim B^{2}$ in the high-field limit, where the WL phase coherence is broken, while for in-plane orientation MR is sublinear with $B$, which gives a tendency for saturation as $B \rightarrow \infty$. Such a behavior is characteristic for open and closed orbits, respectively, expected for a cylinderlike FS [47].

Such a reduced, cylindrical Fermi surface is a characteristic feature of 2D systems, as high-mobility ultrathin films and heterostructures [48-51] and unparalleled cases of pure $\mathrm{Bi}$ and graphene, where the FS covers only $10^{-5}$ and $10^{-6}$ of the Brillouin zone, respectively [52-55]. The renormalization of the $\mathrm{Na}_{1.5}\left(\mathrm{PO}_{2}\right)_{4}\left(\mathrm{WO}_{3}\right)_{20}$ electronic structure driven by the charge density wave, causing the lowering of the Fermi wave vectors, brings this bulk system close to a natural superlattice, with a reduced number of carriers and/or reduced Fermi energy, which promotes the quantum phenomena.

In conclusion, we report the structural and transport properties of the monophosphate tungsten bronze $\mathrm{Na}_{1.5}\left(\mathrm{PO}_{2}\right)_{4}\left(\mathrm{WO}_{3}\right)_{20}$. We demonstrate that this single crystal shows multilayered $2 \mathrm{D}$ weak localization effects. As soon as the dephasing by the magnetic field dominates and breaks the WL contribution, there is a sudden crossover to the large quantum oscillation regime. This shows that natural crystals with $2 \mathrm{D}$ characteristics and reduced carrier density possibly due to a CDW condensation can be used as promising systems for investigating enhanced quantum transport properties.

A.P. would like to thank Thierry Klein and Andrea Gauzzi for valuable remarks on the competition between weak localization and quantum oscillation conditions which motivated our analysis. Financial support by the ANR Projects No. ANR-18-CE92-0014 and No. ANR-11-BS040004 is gratefully acknowledged. Work in Spain was supported by MICIU (PGC2018-096955-B-C44 and PGC2018093863-B-C22), MINECO through the Severo Ochoa (SEV2015-0496) and Maria de Maeztu (MDM-2017-0767) Programs, and the Generalitat de Catalunya (2017SGR1506 and 2017SGR1289). E.D.-N. was supported by the project NanoCent-Nanomaterials center for advanced applications, Project No. CZ.02.1.01/0.0/0.0/15_003/0000485, financed by the ERDF. 
[1] Y. A. Kharkov and O. P. Sushkov, Sci. Rep. 6, 34551 (2016).

[2] O. Cyr-Choinière, S. Badoux, G. Grissonnanche, B. Michon, S. A. A. Afshar, S. Fortier, D. LeBoeuf, D. Graf, J. Day, D. A. Bonn, W. N. Hardy, R. Liang, N. Doiron-Leyraud, and L. Taillefer, Phys. Rev. X 7, 031042 (2017).

[3] W. Xie, X. Tang, Y. Yan, Q. Zhang, and T. M. Tritt, J. Appl. Phys. 105, 113713 (2009).

[4] J. Mao, Z. Liu, and Z. Ren, npj Quantum Mater. 1, 16028 (2016).

[5] K. K. Kolincio, R. Daou, O. Pérez, L. Guérin, P. Fertey, and A. Pautrat, Phys. Rev. B 94, 241118(R) (2016).

[6] S. Molina-Valdovinos, J. Martínez-Rivera, N. Moreno-Cabrera, and I. Rodríguez-Vargas, Physica E 101, 188 (2018).

[7] P. Monceau, Adv. Phys. 61, 325 (2012).

[8] G. Grüner, Rev. Mod. Phys. 60, 1129 (1988).

[9] S. Yasuzuka, Y. Okajima, S. Tanda, K. Yamaya, N. Takeshita, and N. Môri, Phys. Rev. B 60, 4406 (1999).

[10] C. Schlenker, J. Dumas, C. Escribe-filippini, H. Guyot, J. Marcus, and G. Fourcaudot, Philos. Mag. B 52, 643 (1985).

[11] T. A. Sedrakyan and M. E. Raikh, Phys. Rev. Lett. 100, 106806 (2008).

[12] L. Wang, M. Yin, A. Khan, S. Muhtadi, F. Asif, E. S. Choi, and T. Datta, Phys. Rev. Applied 9, 024006 (2018).

[13] V. Tayari, N. Hemsworth, I. Fakih, A. Favron, E. Gaufrès, G. Gervais, R. Martel, and T. Szkopek, Nat. Commun. 6, 7702 (2015).

[14] F. Sarcan, S. Mutlu, E. Cokduygulular, O. Donmez, A. Erol, J. Puustinen, and M. Guina, Semicond. Sci. Technol. 33, 064003 (2018)

[15] W. A. d. Heer, C. Berger, X. Wu, M. Sprinkle, Y. Hu, M. Ruan, J. A. Stroscio, P. N. First, R. Haddon, B. Piot, C. Faugeras, M. Potemski, and J.-S. Moon, J. Phys. D 43, 374007 (2010).

[16] B. Jabakhanji, D. Kazazis, W. Desrat, A. Michon, M. Portail, and B. Jouault, Phys. Rev. B 90, 035423 (2014).

[17] P. Roussel, O. Pérez, and P. Labbé, Acta Crystallogr., Sect. B 57, 603 (2001).

[18] P. Foury-Leylekian and J.-P. Pouget, Solid State Sci. 4, 387 (2002).

[19] P. Roussel, P. Labbé, H. Leligny, D. Groult, P. Foury-Leylekian, and J. P. Pouget, Phys. Rev. B 62, 176 (2000).

[20] B. Domengés, M. Hervieu, B. Raveau, and M. O’Keeffe, J. Solid State Chem. 72, 155 (1988).

[21] E. Canadell and M.-H. Whangbo, Phys. Rev. B 43, 1894 (1991).

[22] K. Kolincio, O. Pérez, S. Hébert, P. Fertey, and A. Pautrat, Phys. Rev. B 93, 235126 (2016).

[23] P. Roussel, A. Masset, B. Domengés, A. Maignan, D. Groult, and P. Labbé, J. Solid State Chem. 139, 362 (1998).

[24] See Supplemental Material at http://link.aps.org/supplemental/ 10.1103/PhysRevB.101.161117 for details of the structural analysis and the computational methods, which contains Refs. [23,56-67].

[25] A. Girard, T. Nguyen-Thanh, S. M. Souliou, M. Stekiel, W. Morgenroth, L. Paolasini, A. Minelli, D. Gambetti, B. Winkler, and A. Bosak, J. Synchrotron Rad. 26, 272 (2019).

[26] E. Wang, M. Greenblatt, I. E.-I. Rachidi, E. Canadell, and M.-H. Whangbo, J. Solid State Chem. 81, 173 (1989).

[27] G. Bergmann, Phys. Rep. 107, 1 (1984).

[28] P. A. Lee and T. V. Ramakrishnan, Rev. Mod. Phys. 57, 287 (1985).
[29] J. K. Moyle, J. T. Cheung, and N. P. Ong, Phys. Rev. B 35, 5639 (1987).

[30] H. L. Stormer, J. P. Eisenstein, A. C. Gossard, W. Wiegmann, and K. Baldwin, Phys. Rev. Lett. 56, 85 (1986).

[31] W. Szott, C. Jedrzejek, and W. P. Kirk, Phys. Rev. Lett. 63, 1980 (1989).

[32] W. Szott, C. Jedrzejek, and W. P. Kirk, Phys. Rev. B 40, 1790 (1989).

[33] W. Szott, C. Jedrzejek, and W. P. Kirk, Phys. Rev. B 45, 3565 (1992).

[34] B. Domengés, F. Studer, and B. Raveau, Mater. Res. Bull. 18, 669 (1983).

[35] J. S. Meyer, A. Altland, and B. L. Altshuler, Phys. Rev. Lett. 89, 206601 (2002).

[36] S. Hikami, A. I. Larkin, and Y. Nagaoka, Prog. Theor. Phys. 63, 707 (1980).

[37] M. Lang, L. He, X. Kou, P. Upadhyaya, Y. Fan, H. Chu, Y. Jiang, J. H. Bardarson, W. Jiang, E. S. Choi, Y. Wang, N.-C. Yeh, J. Moore, and K. L. Wang, Nano Lett. 13, 48 (2013).

[38] M. Liu, J. Zhang, C.-Z. Chang, Z. Zhang, X. Feng, K. Li, K. He, L.-1. Wang, X. Chen, X. Dai, Z. Fang, Q.-K. Xue, X. Ma, and Y. Wang, Phys. Rev. Lett. 108, 036805 (2012).

[39] P. Sheng, Y. Sakuraba, Y.-C. Lau, S. Takahashi, S. Mitani, and M. Hayashi, Sci. Adv. 3, e1701503 (2017).

[40] E. J. McCormick, M. J. Newburger, Y. K. Luo, K. M. McCreary, S. Singh, I. B. Martin, E. J. Cichewicz, B. T. Jonker, and R. K. Kawakami, 2D Mater. 5, 011010 (2017).

[41] S. Thomas, D. J. Kim, S. B. Chung, T. Grant, Z. Fisk, and J. Xia, Phys. Rev. B 94, 205114 (2016).

[42] I. M. Lifshitz and A. M. Kosevich, Zh. Eksp. Teor. Fiz. 29, 730 (1956) [Sov. Phys. JETP 2, 636 (1956)].

[43] D. Shoenberg, Magnetic Oscillations in Metals, Cambridge Monographs on Physics (Cambridge University Press, Cambridge, U.K., 1984).

[44] A. Ottolenghi and J.-P. Pouget, J. Phys. I France 6, 1059 (1996).

[45] G. M. Minkov, O. E. Rut, A. V. Germanenko, A. A. Sherstobitov, B. N. Zvonkov, E. A. Uskova, and A. A. Birukov, Phys. Rev. B 65, 235322 (2002).

[46] Y. A. Pusep, M. B. Ribeiro, H. Arakaki, C. A. de Souza, P. A. Zanello, S. Malzer, and G. H. Döhler, J. Phys.: Condens. Matter 16, 2447 (2004).

[47] A. Pippard, Magnetoresistance in Metals, Cambridge Studies in Low Temperature Physics (Cambridge University Press, Cambridge, U.K., 2009).

[48] J. Wu, H. Yuan, M. Meng, C. Chen, Y. Sun, Z. Chen, W. Dang, C. Tan, Y. Liu, J. Yin, Y. Zhou, S. Huang, H. Q. Xu, Y. Cui, H. Y. Hwang, Z. Liu, Y. Chen, B. Yan, and H. Peng, Nat. Nanotechnol. 12, 530 (2017).

[49] N. Gillgren, D. Wickramaratne, Y. Shi, T. Espiritu, J. Yang, J. Hu, J. Wei, X. Liu, Z. Mao, K. Watanabe, T. Taniguchi, M. Bockrath, Y. Barlas, R. K. Lake, and C. N. Lau, 2D Mater. 2, 011001 (2014).

[50] Y. Kozuka, M. Kim, C. Bell, B. G. Kim, Y. Hikita, and H. Y. Hwang, Nature (London) 462, 487 (2009).

[51] L. Li, G. J. Ye, V. Tran, R. Fei, G. Chen, H. Wang, J. Wang, K. Watanabe, T. Taniguchi, L. Yang, X. H. Chen, and Y. Zhang, Nat. Nanotechnol. 10, 608 (2015).

[52] D. E. Soule, J. W. McClure, and L. B. Smith, Phys. Rev. 134, A453 (1964).

[53] R. N. Bhargava, Phys. Rev. 156, 785 (1967). 
[54] K. Behnia, L. Balicas, and Y. Kopelevich, Science 317, 1729 (2007).

[55] V. Édel'man, Adv. Phys. 25, 555 (1976).

[56] V. Petř́iček, M. Dušek, and L. Palatinus, Z. Kristallogr. - Cryst. Mater. 229, 345 (2014).

[57] L. Palatinus and G. Chapuis, J. Appl. Crystallogr. 40, 786 (2007).

[58] P. Hohenberg and W. Kohn, Phys. Rev. 136, B864 (1964).

[59] W. Kohn and L. J. Sham, Phys. Rev. 140, A1133 (1965).

[60] J. P. Perdew, K. Burke, and M. Ernzerhof, Phys. Rev. Lett. 77, 3865 (1996).

[61] J. M. Soler, E. Artacho, J. D. Gale, A. García, J. Junquera, P. Ordejón, and D. Sánchez-Portal, J. Phys.: Condens. Matter 14, 2745 (2002).
[62] E. Artacho, E. Anglada, O. Diéguez, J. D. Gale, A. García, J. Junquera, R. M. Martin, P. Ordejón, J. M. Pruneda, D. SánchezPortal, and J. M. Soler, J. Phys.: Condens. Matter 20, 064208 (2008).

[63] N. Troullier and J. L. Martins, Phys. Rev. B 43, 1993 (1991).

[64] L. Kleinman and D. M. Bylander, Phys. Rev. Lett. 48, 1425 (1982).

[65] E. Artacho, D. Sánchez-Portal, P. Ordejón, A. García, and J. M. Soler, Phys. Status Solidi B 215, 809 (1999).

[66] H. J. Monkhorst and J. D. Pack, Phys. Rev. B 13, 5188 (1976).

[67] R. Poloni, J. Íñiguez, A. García, and E. Canadell, J. Phys.: Condens. Matter 22, 415401 (2010). 